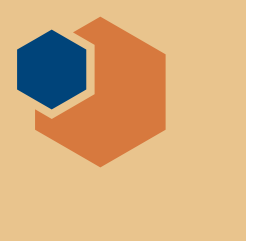

\title{
The long and winding path to nuclear fusion: The ultimate energy source
}

$\mathrm{O}^{\prime}$ October 31, 1952, the detonation of the "Ivy Mike" experimental thermonuclear device at the Enewetak Atoll became the first-ever use of nuclear fusion on the surface of the Earth. The 10.4 megaton explosion, which capitalized on the immense energy release associated with fusion of hydrogen atoms into helium, paved the way for new weapons with unparalleled destructive powers. However, harnessing this energy in a controllable fashion for electricity production has proven to be much more elusive to researchers.

Success in this endeavor would bestow the ultimate source of energy upon the world: according to a recent General Atomics news release, a fusion power plant could use only $5 \mathrm{~kg}$ of hydrogen to generate the energy equivalent of: 18,750 tons of coal; 56,000 barrels of oil; or 755 acres of solar panels. Its operation would neither produce significant radioactive waste quantities nor pose any meltdown risks. Coupled with zero greenhouse gas emissions, this technological breakthrough could transform every aspect of current civilization.

The Second United Nations International Conference on the Peaceful Uses of Atomic Energy held in 1958 has been noted to be the launching point for numerous international collaborative efforts aimed at breaking through this technological challenge for fusion energy. Even after 60 years, the humorous "nuclear fusion is 30 years away, and it always will be" refrain reflects the general public's assessment of this energy source's prospects. But the confluence of multinational research projects reaching key milestones, ongoing federally funded initiatives, and substantial private sector industry involvement offers a chance of finally breaking this paradigm.

As summarized in the 2019 US Congressional Research Service report (CRS) entitled Advanced Nuclear Reactors, the overarching strategy for any fusion power-generating technology focuses on generating a plasma (ionized gas) of hydrogen isotopes. Most common efforts to accomplish this fall into one of two conceptual designs. The first is the tokamak, which is in the form of a torus (ring-shaped donut) that uses external magnetic fields to confine plasma. The second is a stellarator that uses a helical shape to accomplish the same goal. Once either one of these designs can heat plasma above $100 \mathrm{M}^{\circ} \mathrm{C}$, fusion

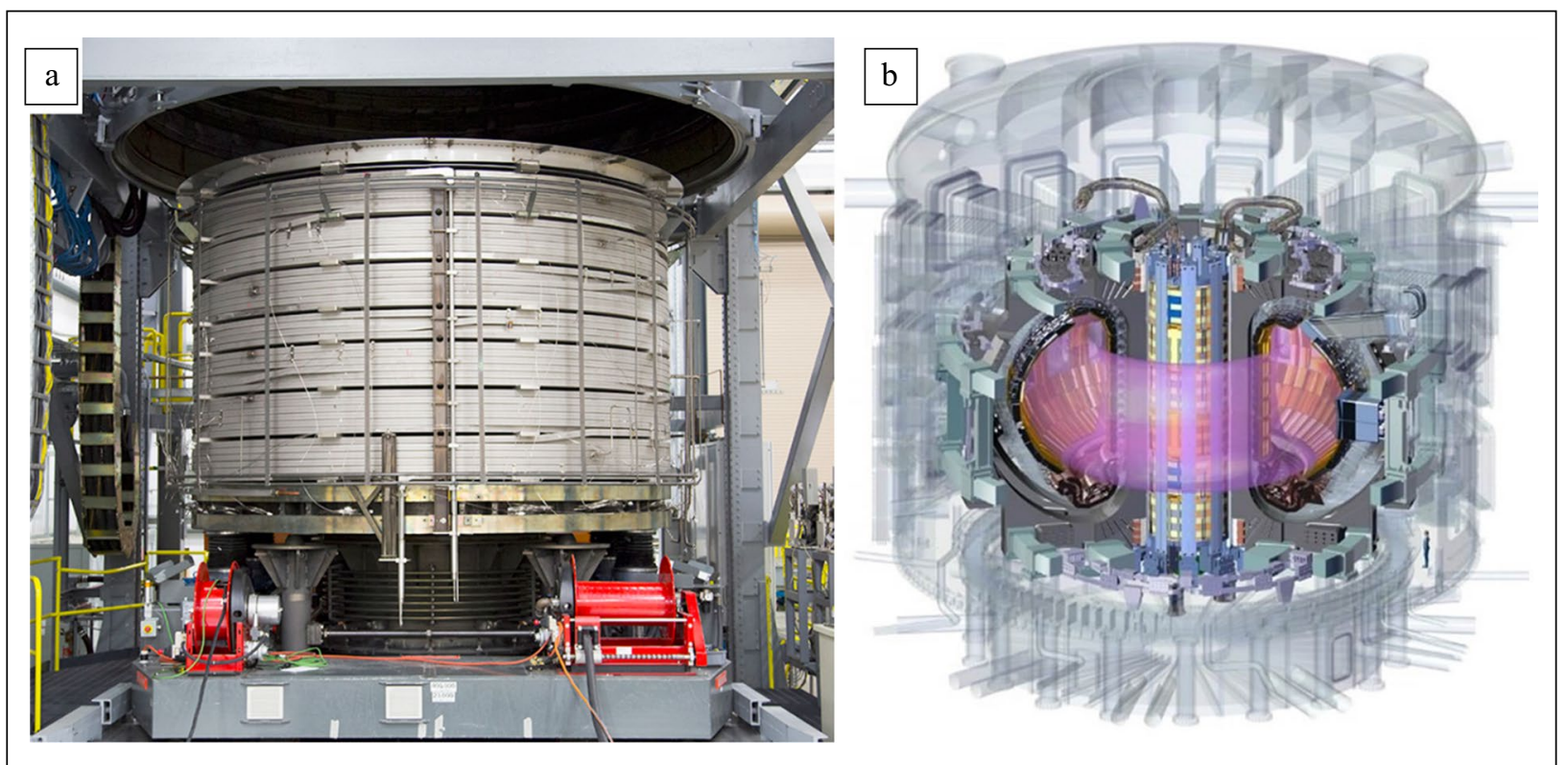

(a) In June 2021, General Atomics shipped the first Central Solenoid to the International Thermonuclear Experimental Reactor (ITER). This 250,000-pound magnet consists of a 560-turn coil comprised of niobium-tin alloy that winds for over 26 miles and generates a 13 -Tesla magnetic field $(280,000$ times stronger than the one produced by the Earth). Credit: General Atomics. (b) Schematic of the proposed ITER tokamak. Credit: ITER. 
of atoms into helium facilitates the required energy release. The crucial element of the technology is the condition of "burning plasma," at which point the system outputs at least as much energy as is required to generate and maintain the plasma. According to a National Academy of Sciences (NAS) 2021 report, to date, no fusion reactor has met this goal: the highest-known ratio of fusion power to input power stands at $67 \%$ (this number must exceed $100 \%$ for fusion reactors to produce net energy).

Materials science breakthroughs remain essential toward development of "burning plasma" fusion concepts. A 2018 report by the US Department of Energy (DOE) Fusion Energy Sciences Advisory Committee identified the essential roles of superconductors and advanced materials and manufacturing in transforming fusion power systems and improving the commercial viability of this technology. Its 2020 followup included development of materials that operate in extreme environments as a prerequisite for development of a fusion power-generating pilot plant by 2040. Materials that comprise components of this reactor must withstand the extreme heat of the plasma that they contain, precisely conform to highcurvature toroid and helical surfaces, and survive neutron radiation damage emanating from the plasma. A 2020 American Physical Society report on fusion, which, among its contributors, included subject matter experts from the Fusion Materials Program at Oak Ridge National Laboratory (ORNL), included rapid expansion of the fusion materials and technology program as one of its key recommendations. Guinevere Shaw, a DOE Office of Science Fusion Energy Sciences program manager, stressed the importance of the development of structural materials for the first wall and divertor that can withstand the harsh plasma environment. Shaw highlighted the importance of a collaborative fusion materials and technology program that is required to develop many of the integrated and complex fusion components needed for a future fusion device.
Fusion reactor fuel is another crucial component that is integral to the ITER project and subsequent domestic (US) pilot plant development. The NAS 2021 report states that a mixture of deuterium (hydrogen isotope with one additional neutron) and tritium (two additional neutrons) is the optimal precursor to "burning plasma" in tokamaks. These isotopes require lower temperatures than neutron-less hydrogen, or any other combination of elements, to achieve fusion. Although the amount directly consumed by a fusion reaction is low (a 500-megawatt plant would only consume $75 \mathrm{~g}$ per day), escape of these light atoms from the plasma increases daily expected hydrogen isotope requirements to over $7500 \mathrm{~g}$ for such a system. A 2001 CRS report on tritium production highlighted the approach used at the Savannah River Site in the United States: neutrons generated by a nuclear reactor strike lithium targets and generate the required tritium atoms. In 2017 , a federal record of decision issued by the Tennessee Valley Authority outlined its production of the isotope using light water reactors and irradiation of specialized material rods. Both methods produce small quantities of tritium, and a 2017 Defense News (US) article warned of an upcoming shortage of this material due to limited domestic manufacturing capabilities. However, tritium's dual use in production of nuclear weapons raises proliferation concerns, and compliance with international treaties will challenge efforts to ramp up its manufacturing.

Owing to the immense scope of the technological challenge, fusion research has, historically, been carried out in the form of multinational collaborations. These efforts leverage worldwide human capital resources of mathematicians, physicists, engineers, materials scientists, and other subject matter experts. The International Atomic Energy Agency has already coordinated several multinational projects, such as the 1979 International Tokamak Reactor and the Germany-based Axially Symmetric Divertor Experiment tokamak experiment of the early 1980s.
In 1988, these initial efforts culminated in the formation of the International Thermonuclear Experimental Reactor (ITER). This project aims to construct a facility that successfully yields "burning plasma" experiments. Starting in 2007, China, the European Union, India, Japan, Russia, South Korea, and the United States commenced its construction in Southern France. DOE manages the US role in ITER and primarily manages relevant research activities through ORNL, the Princeton Plasma Physics Laboratory, and the Savannah River National Laboratory.

DOE oversees and funds most nuclear fusion research in the United States. Its fiscal year (FY) 2019 appropriation, which includes the ITER funds, allocated USD\$574 million to fusion research-15\% of the entire R\&D portfolio. The department authorized USD\$643 million in FY 2020 and is proposing a modest increase to USD\$675 million in FY 2022. High-energy physics research, which complements fusion work, received USD \$1.046 billion in FY 2021. Out of the fusion energy portfolio, the United States allocated USD \$242 million annually to the ITER project in both FY 2020 and FY 2021. According to a 2021 US CRC report on Energy and Water Development Appropriations the United States shoulders $9 \%$ of the total project cost, which will, cumulatively, consume as much as USD $\$ 6.5$ billion of the US federal budget once it is complete.

Continued engagement in the ITER program has received periodic pushback and questioning from lawmakers, who doubt the effort's charges of successfully bringing nuclear fusion to the domestic electrical grid. Federal funding of the US contribution to the program was cut across several budget cycles within the last decade. In 2016, the US Senate Appropriations Committee considered ending US participation in the program altogether. Nevertheless, the program has evaded most budget cuts, and, from FY 2019 to FY 2020, annual allocation of funds for the US 
contribution to ITER increased from USD\$132 million to USD\$242 million; funding for FY 2021 remained flat. Although the project is headquartered in France, ITER has an expansive scope in the United States. Of note, it has awarded contracts to industry (USD\$700 million), academia (USD\$26 million), and DOE National Laboratories (USD\$489 million) across 46 states. Moreover, this project supports 1600 direct and indirect jobs in the United States.

The US ITER program brief estimates the project as approximately $70 \%$ complete. If funding levels remain steady, the constructed facility will commence experiments in 2025 and aim to generate "burning plasma" no earlier than 2035. The country's return on its investment will arrive as $100 \%$ of scientific data and intellectual property developed during the program. Even if the ITER project succeeds, the United States will only be able to benefit from the program if it can onshore construction of "burning plasma" facilities and advance their development into nuclear fusion reactor prototypes. Both the 2019 and 2021 NAS reports on fusion research strongly recommend continued US participation in the ITER project and its funding, because a go-at-it-alone fusion research project would, according to these studies, almost certainly preclude domestic deployment of this technology at any scale in the 2040s.

In addition to ITER, other large-scale fundamental endeavors persistently attempt to tackle the fundamental problem of heating and sustaining "burning plasma." DOE's Fusion Energy Sciences (FES) division also includes the National Spherical Torus Experiment (NSTX). At its location in the Princeton Plasma Physics Laboratory, researchers are investigating an alternative geometrical configuration of the tokamak and generated plasma. The facility is undergoing a large upgrade that aims to position the NSTX as a viable fusion reactor pilot plant. The joint DOE-General Atomics DIII-D Program operates as an open international user facility with
106 participating institutions and over 600 research collaborators. Its National Fusion Facility developed the firstknown noncircular cross-section ("Doublet") tokamak in the 1960 s, and ongoing research aims to further advance this technology's capabilities.

Government facilities outside of the FES are also involved in these activities. Since 2010, the National Ignition Facility (NIF) at the Lawrence Livermore National Laboratory has used a network of 192 laser beams to fire nanosecondlong pulses at small targets to achieve this effect. A 2020 Science article reports that, since then, technological advancements in both laser and target configurations have pushed maximum achievable temperature from $11 \mathrm{M}^{\circ} \mathrm{C}$ to over $50 \mathrm{M}^{\circ} \mathrm{C}$. Most recently, the laboratory announced that its August 2021 ignition experiment achieved a yield of 1.3 megajoules. However, these metrics fall far short of the plasma ignition conditions: 1.65 megajoules, and temperatures of over $100 \mathrm{M}^{\circ} \mathrm{C}$, are needed to achieve sustainable fusion. Although the facility has delivered fundamental physical insights and provided nuclear arsenal safety and stewardship analysis to the National Nuclear Security Administration (the facility's funder), NIF's laser experiments in support of plasma ignition-dedicated work have fallen from $60 \%$ of all total fired pulses at NIF in 2012 to $30 \%$ in 2020. Multiple fundamental hurdles remain, and, with an ongoing review of its progress, the facility's role in nuclear fusion research is uncertain.

Private industry is also stepping up its involvement and investment in this technology area. The 2020 CRS report on Advanced Nuclear Reactors mentions AGNI Energy, Brillouin Energy, Commonwealth Fusion Systems, General Atomics, Helion Energy, HyperV Technologies, Lawrenceville Plasma Physics, Lockheed Martin, MagnetoInertial Fusion Technologies, NumerEx, and TAE Technologies as entities with ongoing fusion projects. The Fusion Industry Association, which was established in 2018, is 25-member strong and pursues a broad set of initiatives to advocate on behalf of this technology and advance its development. A recent article in the Canary Media estimated that fusion has attracted over USD\$2 billion of private investment and venture capital funding in the last few years. Most recently, Helion Energy announced that its test reactor achieved the $100 \mathrm{M}^{\circ} \mathrm{C}$ milestone. In an interview with MRS Bulletin, representatives of the company said that $100 \mathrm{M}^{\circ} \mathrm{C}$ "is the temperature required for a commercial fusion system. Achieving this milestone demonstrates that Helion's non-ignition, pulsed approach has a clear path to generating electricity from fusion."

Ultimately, these efforts aim to develop nuclear fusion pilot plant prototypes and bring this energy source into production and commercialization. To accelerate these efforts, DOE established the Innovation Network for Fusion Energy (or INFUSE) that funds public-private research partnerships with USD $\$ 50,000-U S D \$ 500,000$ awards and includes a $20 \%$ cost share for industry partners. This program, which announced its second round of awards in September 2020, focuses on magnets and fueling, materials, diagnostics, modeling, and experimental capabilities. DOE's Advanced Research Projects Agency announced two fusion programs in 2020: Galvanizing Advances in Market-aligned fusion for an Overabundance of Watts (GAMOW) and Breakthroughs Enabling THermonuclear-fusion Energy (BETHE). These efforts pursue cost-effective powergenerating technology; BETHE aims to bring fusion power plant capital costs below USD $\$ 2$ billion. Shaw highlighted these programs and the opportunities that they provide to interested industry partners and researchers to further technologies that could impact development of fusion components needed for future fusion devices.

While every scientific challenge carries an inherent degree of risk, success remains especially uncertain for fusion power research. In a 2018 interview with Science|Business, the 
head of the ITER project admitted that he will not live to see the benefits of this endeavor. Nevertheless, recently reported breakthroughs suggest that this work remains promising: the Chinese Academy of Sciences reported in May 2021 that its HT-7U Experimental Advanced Superconducting
Tokamak sustained plasma at $120 \mathrm{M}^{\circ} \mathrm{C}$ for $101 \mathrm{~s}$. Although this success is an unprecedented milestone, the decadesold effort to realize this ultimate energy source is, by most accounts, still multiple decades away from putting fusion power plant-produced electricity onto the power grid. The grand challenge of developing fusion power is deeply rooted in international collaboration, and its eventual success is, inevitably, contingent on large-scale, sustained cooperation between the scientists, governments, and entrepreneurs around the world.

Boris Dyatkin

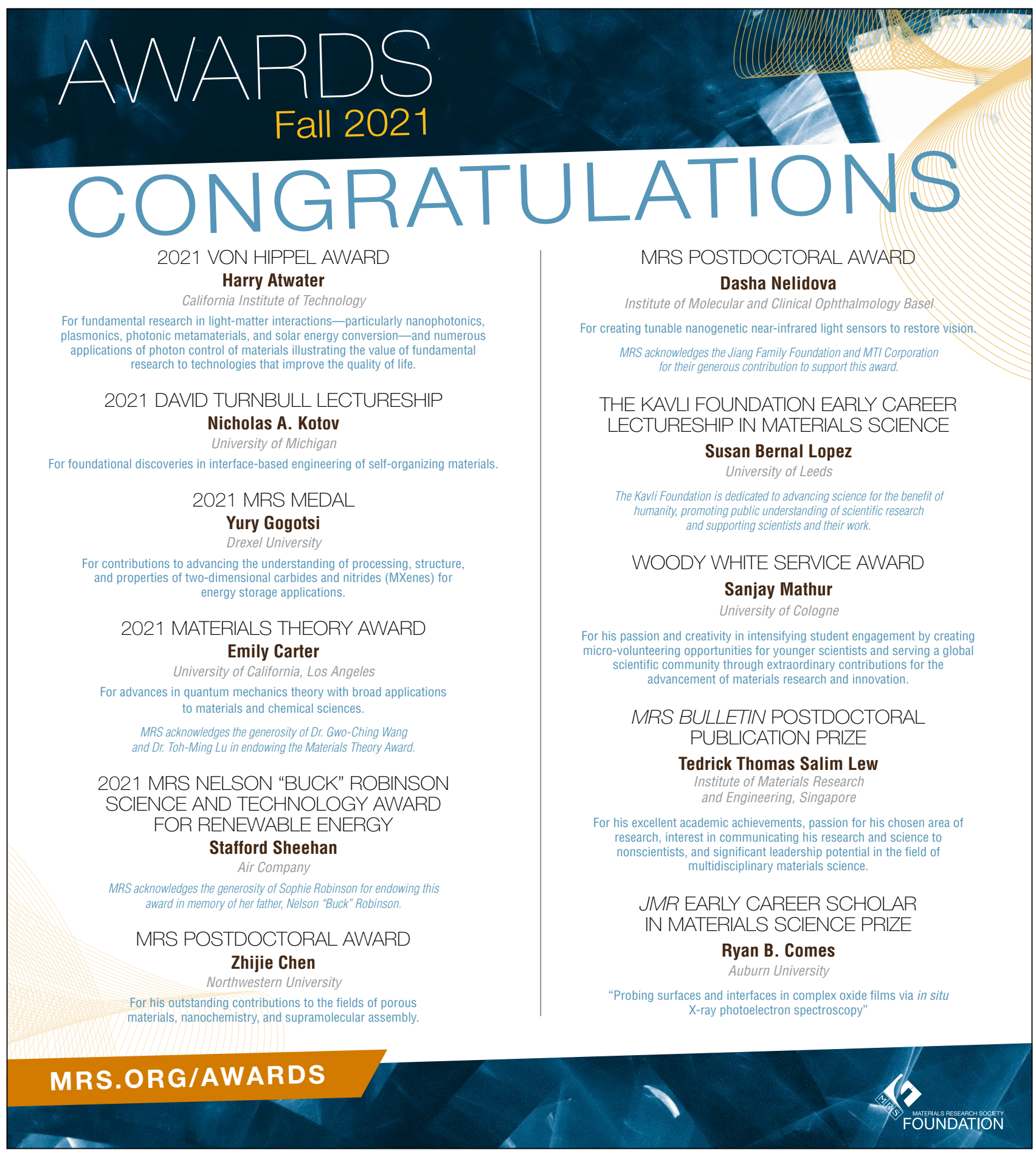

